\title{
Fundamentals of New Effective System to Accelerate Language Acquisition Using Visual Approach
}

\author{
Boyd L. Bradbury, Imad H. Tahini, and Alex K. Dadykin
}

\begin{abstract}
Increased migration of individuals from one country to another poses the challenge of accelerating improved language acquisition while reducing the cost of language training. This work strives to find new ways to improve communication and understanding, including improved quality and a reduced cost of training, as well as new ways of learning and skills training, mainly for adult learners. The use of the visual approach and the visual model, which is based on this approach, and the use of the system analysis tools and instructional technology provide new and encouraging results. This research has led to the development of a training system that utilizes language formation processes and language skill building.
\end{abstract}

Index Terms-Continuous evaluation, interactive speech trainers, learning management system (LMS), structural visual method (SVM), visual models.

\section{INTRODUCTION}

Large-scale emigration of people from one country to another requires education and socialization of immigrants, which is difficult, if not infeasible, in the traditional education system, which was created long ago for very different purposes. Demographic changes necessitate educational change. As an example, United States (US) Census data reveal that the percentage of individuals in the US who spoke non-English only rose from 11 percent in 1980 to 20.3 percent in 2010 [1].

There has been a development of alternative ways of learning, including those that have emerged through technology, such as online learning. The last couple of decades have resulted in rapid growth within the area of technology-assisted learning. Many companies and start-ups have created programs, applications and services for training and educational process management within organizations. Product development has been sufficient in coping with the automation of routine processes of knowledge transfer in the field of theoretical training. However, in practical skills training, including language training, there has been little progress for a number of reasons. For example, complex interdisciplinary developments require coordinated work of specialists in various branches of knowledge and the use of different terminology, methodologies and models within the subject fields. This coordination makes it challenging to

Manuscript received November 10, 2017; revised May 18, 2018.

B. L. Bradbury is with the Minnesota State University Moorhead, USA (e-mail: bradbury@mnstate.edu).

A. K. Dadykin is with the Belarusian National Technical University, Minsk, Belarus (e-mail: alex_05_07@rambler.ru).

I. H. Tahini is with the Belarusian National Technical University, Beirut, Lebanon (e-mail: imad.tahini@live.com). work together, which results in outdated methods within established systems that utilize technology. Simply put, teachers are not using the technological capacities of modern systems to the extent possible in light of improved discoveries and developments.

The proposed Structural-Visual Method [2] offers a new approach for the harmonization of terminology in interdisciplinary projects, and it can be used to create new tools for learning skills and foreign languages. The essence of the approach is structured knowledge, which moves from the most basic to the more complex and translating information about the rules (e.g., verb conjugation) of the subject field into a visual form. Development of visual meta-language to non-verbal communication with the teacher-student, human-computer, and human-human systems can help improve training and create a new generation of LMS.

\section{StRUCTURE OF LEARNING MANAGEMENT SYSTEM OF NEW GENERATION}

The basis for the harmonization of terminology, methods, procedures and structures in the process of creating a new generation of LMS involves visual meta-language tools and systems analysis as links between theory and practice, information technology (IT), and processes within the fields of humanities, science and social (Fig. 1).

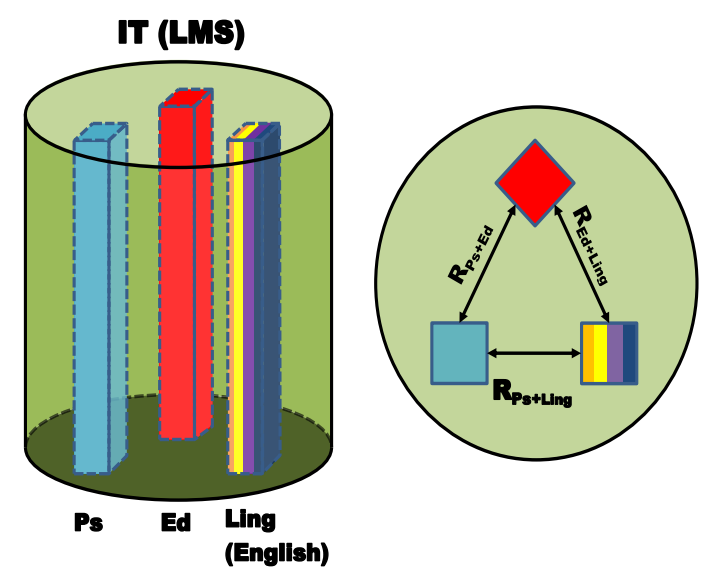

Fig. 1. Structural diagram of LMS (upper level of abstraction), where: Ed education; Ps - psychologic; Ling - linguistic.

The authors recommend a conceptual solution in the form of the creation of a training system [3], which allows the control of the process of language formation and development of language skills for adults. The generalized structure of the new generation LMS is shown in Fig. 2.

This system combines methodical principles that are grounded in the works of Bandura [4], Galperin, [5] and 
Visual Models [6], which create a synergetic effect, both in the initial phase of mastering a foreign language, and at the stage of "barrier to overcome." The use of IT as a tool is essential for ensuring the implementation of learning objectives with continuous monitoring and feedback of the learner's progress in a finite number of steps.

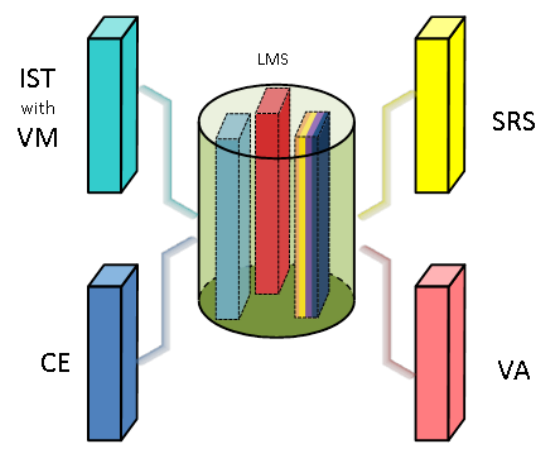

Fig. 2. LMS generalized structure: IST with VM (interactive speech trainers with visual models), SRS (speech recognition system), CE (continuous evaluation), VA (virtual assistant).

The development of the main components of LMS is carried out in three interrelated steps:

1) Further improvement of the SVM by modeling the structure of the developed activities based on the Visual Models.

2) Setting up the subsystem of Continuous Evaluation and the management of the learning process in real time.

3) Creation of a set of Interactive Speech Trainers that correspond to different levels of competency of the students.

\section{INFORMATION-COMMUNICATION MODEL AND MANAGEMENT OF THE EDUCATIONAL PROCESS}

In order to ensure mutual understanding between basic educational principles and information technologies, special importance must be placed on the nexus of terminology, language learning models, and visual topological analysis and recognition as the language structure, pedagogy, and IT come together. An example of such an approach is the information-communicative model, which shows processes in the data-processing and communication systems during language acquisition, but also serves as a model for communication in teacher - student and speaker - listener interaction. This model further involves interpersonal communication, including nonverbal components (Fig. 3).

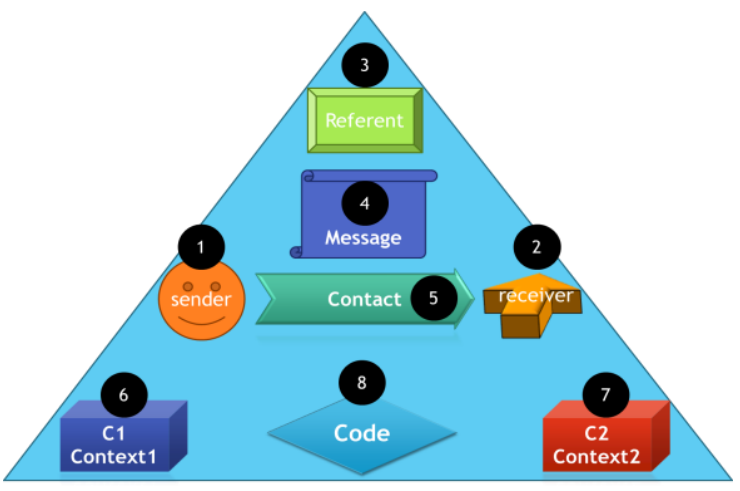

Fig. 3. Information-communicative model.
The Information-communicative model is the link for the introduction of the computer systems of a new generation of educational process management tools that provide not only the structure and transfer of knowledge from teacher to pupil, but also the quantitative and qualitative level feedback for student proficiency with competencies. This model sets parameters and provides feedback regarding the learning curve.

This is especially important in the field of training and retraining of Bandura [4], in which learning occurs from the interaction among the environment, human behavior and properties of personality. At the same time the influence of previous experience leads to the fact that the learning curve, instead of the usual logarithmic growth, becomes distorted with several minimum points, so-called "barriers to overcome".

The use of modern IT in conjunction with the use of effective models for acquiring skills allow reduction or even elimination of the impact of these barriers, thus accelerating training and enhancing success by transferring a synergistic effect to all stages of the formation of language skills, especially "barriers to overcome" (Fig. 4).

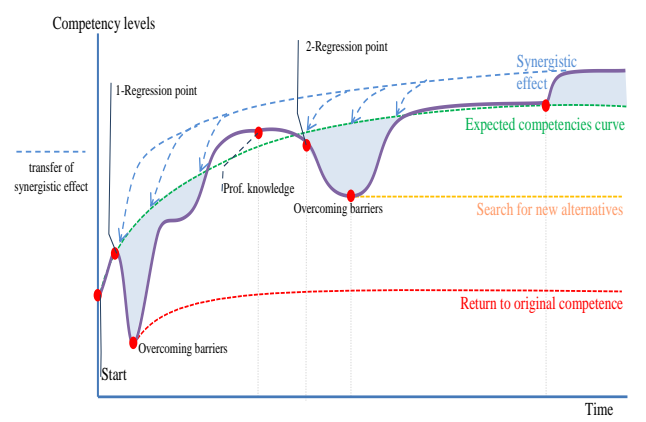

Fig. 4. The learning curve with the transfer of synergistic effect.

The subsystem of Continuous Assessment and Management of the learning process [7] provides the adjustment of the entire system at three levels: speech simulators, the teacher and continuous measurement.

The interface of speech simulators, include elements of gaming, such as socialization and cooperation, to translate the learning process into a modern, intensive, and effective format.

The teacher component involves repositories of training materials and mechanisms for managing the synthesis of training exercises. These repositories have prepared learning activities that provide analysis of the results of the language activity of each trainee.

Continuous measurement involves the control of the teaching process at each specific point of the learning curve or real-time retraining. Individuals learn particular skills within set parameters that are provided. The speed of the exercise is determined not by external instructions or the interface of the training program, but by ability and competency level of each particular student.

\section{Models of Language Teaching}

Providing non-verbal tools for teaching foreign languages, which involves a new form of social interaction and 
communication, is a very promising direction and opens new horizons for the development of effective ways to resolve these problems. The proposed approach allows one to:

1) Separate the knowledge of structure of the language from the words.

2) Move the meaning into the intermediate sign system (maps) in the form of visual models.

3) Then say the same meanings in the target language.

With this as a rough basis of speech activity verbal rules are not used, and the visual structure is called "LingvoMap".

A simple example is shown in Fig. 5 - LingvoMap. It shows the construction of a simple declarative sentence in English for the aspectual-temporal forms, corresponding to the stages of development of a single event in time.

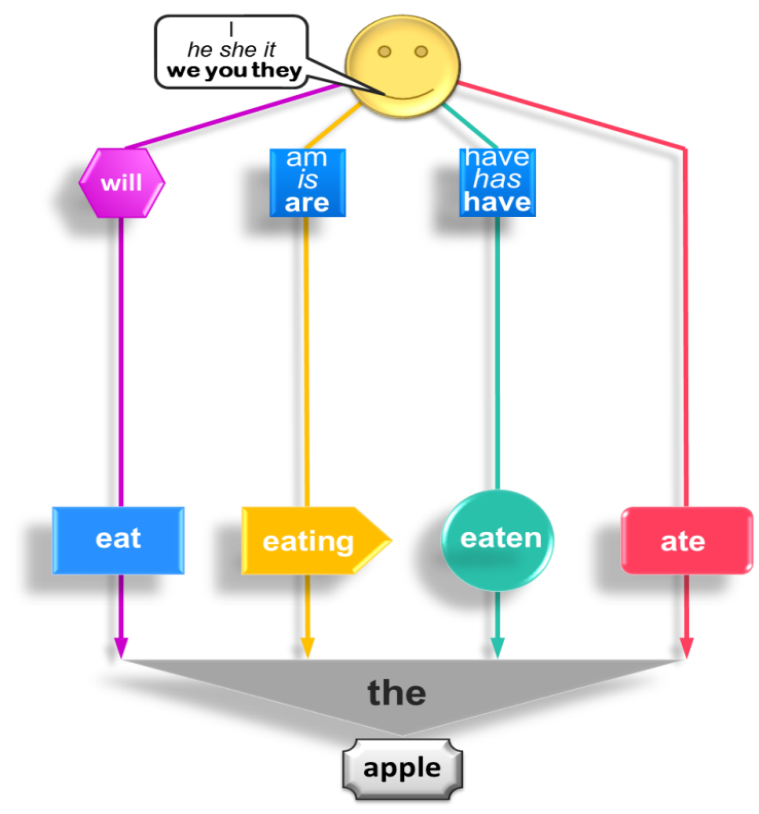

Fig. 5. Model structure of declarative sentence.

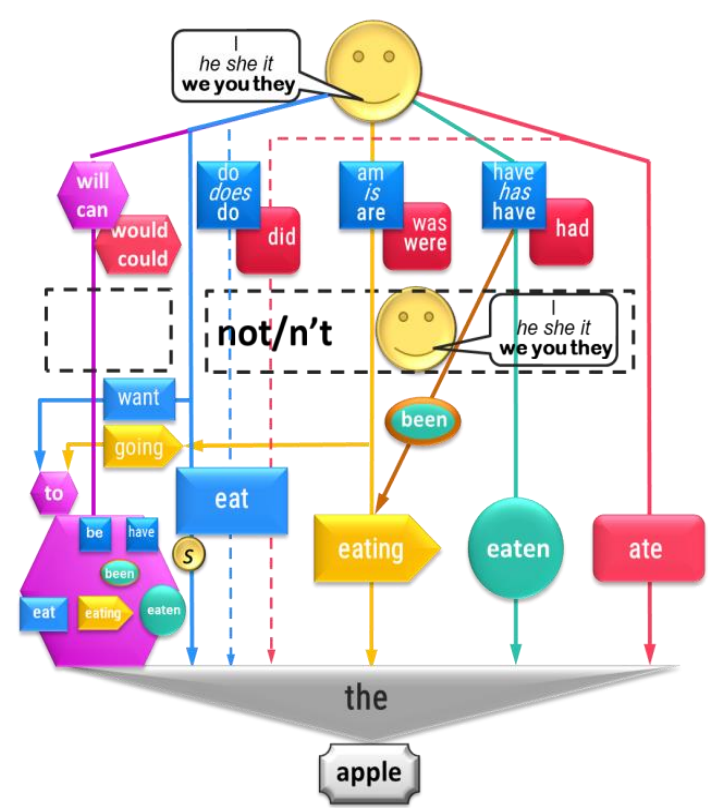

Fig. 6. Full model for all temporary forms.

Grammatical transformations are shown graphically, without words. This allows the student not to think on using the rules of the native language, but directly creates the correct English sentence based on the visual structure.

In the process of language learning, the model becomes more complicated, adapting to the volume of material chosen for a particular lesson. Fig. 6 shows a process for mastering the intermediate models.

To date, we have the full set of visual models for the major structures of the English language, as well as the pre-sets for the Russian and French languages. The development of interactive simulators with the use of speech technologies significantly increases the effectiveness of the application of the Structural-Visual Method.

\section{INTERACTIVE VOICE TRAINERS}

Based on the study mentioned in this topic, it has been shown that there are new methods of learning and that the visual approach and the conversion of sentences to intermediate models, such as maps, is the most effective way to speed up learning. As a result, we are building a program to use images and maps for learner training to acquire language via images of sentence construction. These "Mind Maps," employ visual models and listening, followed by sounding out and repeating the words. All requirements for building the program are in place.

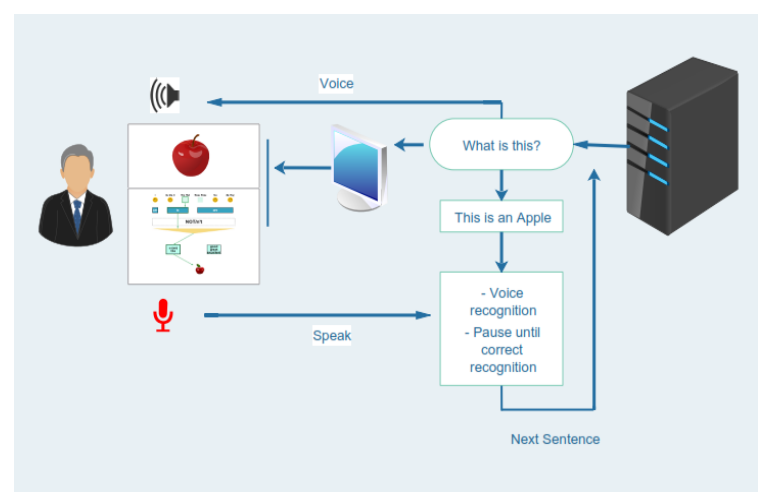

Fig. 7. Functional diagram.

\section{A. Proposed System}

As shown in the Figure 7 above, the training system will do the following:

1) Start a lesson, and get data from database.

2) Set a level and interaction type of the lesson.

3) Set a loop to get each sentence from the database repository of a lesson.

4) Load the image and "LingvoMap" for a sentence.

5) Model a sentence through speed, and then evaluate the correct pronunciation of learner.

6) Use a learning curve to assess learner progress.

7) End a lesson then go to next lesson.

\section{B. Architectural Design}

In Order to achieve the prototype did the following items:

1) Building of Database: For an easy and rapid extraction of information by the user, the knowledge about a particular subject or domain will be stored in a Database.

2) LMS Platform Implementation: The implementation of the LMS Platform used C\# and API for Java script that allowed a connection to the Database, load, and save data. The API model was applied in modern web browsers and code structure responsible for processing the data obtained through the Web Audio reutilized 
interfaces. The API used JavaScript, a language in which there was development of the modules of configuration, the "LingvoMap", data analysis, formation of logarithmic learning curve, and voice processing. We utilized HTML5 that features the Canvas API [8] for programming two-dimensional graphics based on immediate-mode drawing. Both WebGL and the HTML5 Canvas were necessary for developing "LingvoMap".

3) Adaptation of Speech Recognition System to LMS Platform: We propose a mechanism to facilitate the interaction between the user and the platform through an interface that uses voice recognition. In our platform, a template consists of an interactive image and "LingvoMap" representation, which reflects the internal structure of the question from voice recognition. This module would be integrated into the server and also linked to the Automatic Speech Recognition (ASR) system. To achieve this, the following processes are required:

- Voice-to-text: The process used for adapt the voice recognition system is the ASR. We will focus specifically in API Google Speech to Text Service because it is a cloud computing system and does not compromise the performance of the local computer.

- Text-to-Speech: To convert Text to Speech, we propose to use the cloud services of Google. Using Google Translator, however, is a less complex process compared with ASR.

\section{Development Phase}

The development of the proposed visual approach method "LingvoMap" and the course material took place due to the Hannafin Peck [9] instructional design model. Instructional design model helps instructional designers to understand of abstract learning theory and enable real-world application. Fig. 8 below shows the components and model phases. The Hannafin Peck design model is a three-phase process.

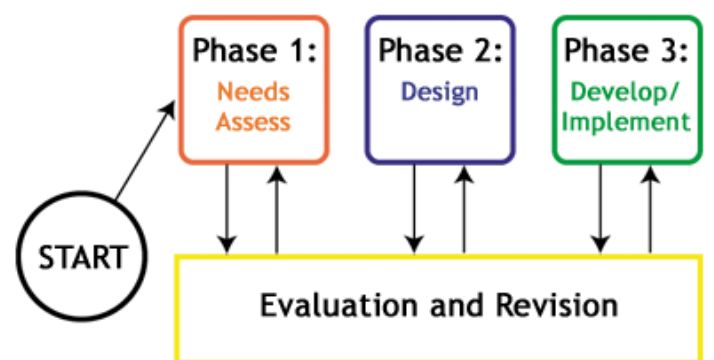

Fig. 8. Design and implementation of educational software model of Hannafin.

Phase I - Needs Assessment: The needs assessment phase consists of determining the program's objectives. It also requires an instructional designer to create reasonable goals for his project.

Phase II - Design: In this phase, one must investigate the overall design, teaching approaches, structure, media, learning theories and technology types that will be included.

Phase III - Development and Implementation: The final phase is development and implementation. The program is put into work, and a decision must be made as to continue or revise it.

\section{DISCUSSION}

The analysis of this study has resulted in the creation of new software (LMS). This LMS is currently under construction now that the requirements of this software have been established. This LMS is at the stage of continued design and development

A preliminary model has been created. To date, approximately 300 students have been part of experimentation associated with the development of this LMS. Encouraging results have been obtained.

Experimental verification of the training materials, test simulators and elements of the proposed approach on a limited group of students showed results similar to those that were obtained by the rapid development of other training techniques for Galperin theory [5]. There was a reduction in training time for performance of specific actions (3-30 times) and an increase in the success of training (10-95\%). These are preliminary results that require additional verification and independent testing.

We need to accumulate experiences by using the LMS simulation (Fig. 9) which will help the technical teams to:

1) Develop simulation models.

2) Optimize designs before construct any new version.

3) Accurately detect weak spots.

4) Efficiently explore multiple design alternatives.

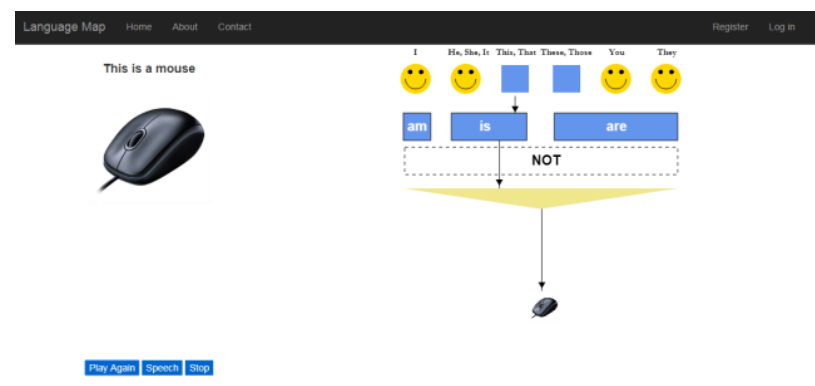

Fig. 9. Interface of IST.

\section{CONCLUSION}

In this research paper, we have explained the concept of software for LMS and clarified new features by using visual technique and a language map. Also, we have reviewed results generated from previous papers regarding this topic.

Currently, this program is being under further development to reach the target result and facilitate learner training on the language. We are updating and developing this program, while conducting experiments to continue to reach an integrated program for teaching all foreign languages. We are focusing on the creation of programs for teaching English, French, Russian and Arabic. After the launch of these programs, we will work to accommodate other languages as well.

\section{REFERENCES}

[1] B. L. Bradbury, “A brief overview of education, demographics, learner management, and instructional approaches for non-English speaking students in the United States and Minnesota State University 
Moorhead," Visiting scholar presentation at Belarussian National Technical University: Minsk, 2017.

[2] A. K. Dadykin and V. A. Dibrova, "Generalized visual model of the structure of the educational process," Modern Trends in Secondary Adult Education: Materials of III International. Scientific-Method. Conf., Minsk, October 21, 2016.

[3] A. K. Dadykin, V. A. Dibrova, and I. H. Tahini, "The visual approach in educational projects," presented at 4th International Conference on Education and Psychological Science (ICEPS 2017), Barcelona, Spain, February 12-14, 2017.

[4] A. Bandura and R. H. Walters, Social learning and Personality Development, New York, Holt, Rinehart, \& Winston, 1963.

[5] P. Y. Galperin, "Psychology of thinking and teaching about the gradual formation of mental actions," Research in the Thinking of Soviet Psychology, Moscow, 1966.

[6] A. K. Dadykin, V. A. Dibrova, and I. H. Tahini, "Visual models of communication and language," in INTED2017 Proc. 11th International Technology, Education and Development Conference, pp. 7124-7132.

[7] A. K. Dadykin, V. A. Dibrova, and I. H. Tahini, "Visual approach and learning process management," in INTED2017 Proc. 11th International Technology, Education and Development Conference, pp. 7062-7071.

[8] S. Fulton and J. Fulton, HTML5 Canvas, O'Reilly Media, Incorporated, 2013.

[9] M. Hannafin and K. Peck, The Design, Development, and Evaluation of Instructional Software, 1st ed. Macmillan Publishing, New York, 1988, ch. 5, pp. 58-78.

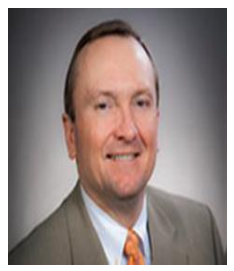

Boyd L. Bradbury is from Minnesota. He is an honors program graduate, and he earned a BA from Bemidji State University, Minnesota, with a major in Spanish, minors in philosophy and anthropology, and an emphasis in international studies in 1988. He earned a MAT in Spanish and an Ed.S. from Mankato State University in Mankato, Minnesota in 1991 and 1996, respectively. He completed the doctoral work at the University of Minnesota - Twin Cities, and he earned a Ph.D. in Genera Education through Capella University in 2005. He served as a high school Spanish teacher, secondary school principal, and superintendent of schools prior to his full-time appointment at Minnesota State University Moorhead (MSUM) in 2007. He is a professor of educational leadership and curriculum and instruction, he coordinates the doctor of education degree, and he served as the dean of the College of Education and Human Services and Graduate Studies at MSUM. Scheduled and recent publications include: The Validity of Utilizing the National School Lunch Program as Proxy for Students' Socioeconomic Status and Its Impact on Academic Performance (Thousand Oaks: CA, Sage Research Case Methods, April 2007), and the Mirage of
Educational Equity among Involuntary Minorities and an Involuntary Majority: A Comparison of Public Education Systems Serving Indigenous Populations in the United States and South Africa (Volume 4, No. 2, Summer 2013, The Roundtable, retrieved from http://spse.us/spse/mpes.html). Research interests include academic achievement, indigenous populations, and pedagogy. Dr. Bradbury received the Bemidji State University Outstanding Alumni Association Award in 2002.

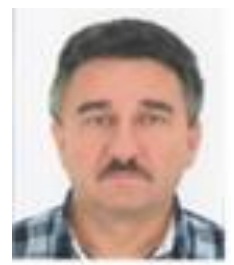

Alex K. Dadykin was born in Minsk. He got the master degree, Ph.D. in automated control systems from Minsk High Engineering Military School of anti-aircraft missile defense graduated with honors, in 1980 and 1989 respectively. In 1991, he was awarded the degree of Candidate of Technical Sciences (Soviet Union), and in 1999 was awarded the academic title of associate professor on "informatics, control and computer engineering" (Republic of Belarus).

From 2001 to 2009, he served as scientific secretary of the Council for the defense of dissertations at the Military Academy of the Republic of Belarus. Currently, he is an associate professor of the Department of Information Systems and Technology at the International Institute of Distance Education and a Chairman of the scientific meeting of the Belarusian National Technical University. He is the developer of four of the State educational standards for higher education for practice-oriented master's degree.

Dr. Dadykin in 2009 was elected Professor of the Academy of Military Sciences of the Russian Federation. He is the author of more than fifty educational-methodical and scientific works, and he has five inventor's certificates. He is the Senior Member of IEDRC and the Member of IEEE His main areas of research are in the field of system analysis, the development of learning management systems, and mobile robotic systems.

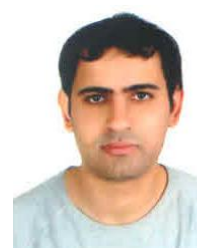

Imad H. Tahini was born in Aita el chaab, Lebanon, March 10, 1981. From 1999 to 2006 he earned a teaching diploma in an applied mathematics option: computer science from Lebanese University faculty of science.From 2011 to 2012 he earned a master's degree in computer science and communication from Arts, Sciences And Technology University in Lebanon. Since 2016, he is a Ph.D. student at Belarusian National Technical University.

From 2006 to 2018, he worked as a programmer, software development team leader and data analyst in HEC, HHC and Servo-pro Companies. He is interested in developing business software and design for a database methodology, and he has business knowledge in accounting and finance. $\mathrm{He}$ is the Member of IEEE. His current research is in the field of learning management systems. 